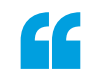

\section{we aim to democratize accelerator technologies to a wide range of applications}

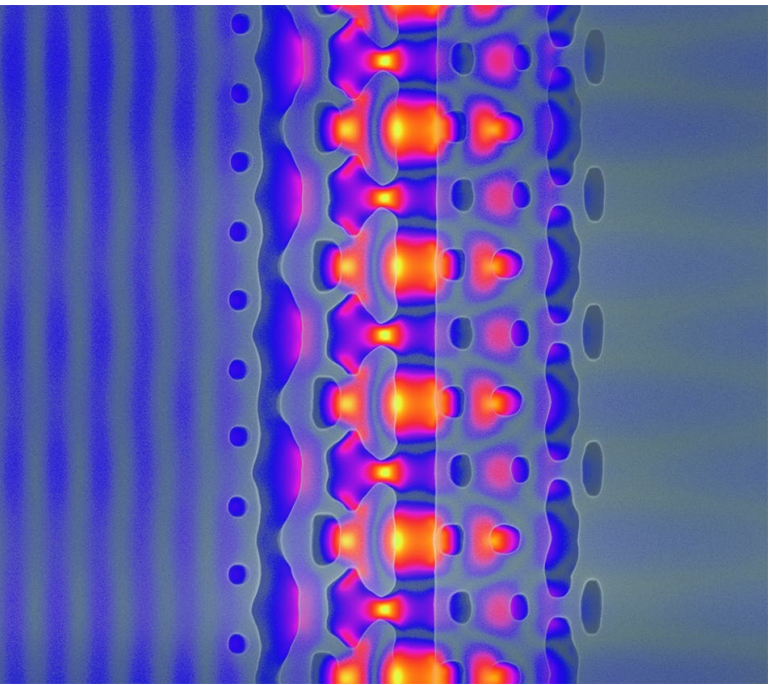

Credit: Image courtesy of N. V. Sapra, Stanford University, USA.
Leveraging a photonic inverse design approach, Jelena Vuckovic and her colleagues created an on-chip laserdriven particle accelerator, as they report in Science, with an energy gain of $0.915 \mathrm{keV}$ over $30 \mu \mathrm{m}$.

Conventional particle accelerators rely on microwaves to provide the kick that accelerates the particles. Because microwave wavelengths are on the centimetre scale, these accelerators are intrinsically big and mostly suited to large-scale facilities.

Now, the widespread availability of powerful pulsed infrared lasers has opened up the possibility to make truly compact particle accelerators based on electromagnetic radiation with a wavelength on the order of microns. "Unfortunately, we can't simply shrink down the conventional metallic accelerators because of the high losses of infrared light in metals," comments Neil Sapra, first author of the study. "Instead, we need to reinvent all the components of an

ACCELERATOR TECHNOLOGIES

\title{
A compact design
}

accelerator in a dielectric material, in which infrared light can travel without attenuation: this type of accelerator is called a dielectric laser accelerator (DLA)."

DLAs are made of periodic structures designed to enable cascaded stages of acceleration. Compact and cost-effective, they can be driven with visible or near-infrared pulsed lasers, which enables a reduction in size of four orders of magnitude compared with conventional accelerators. However, the technology is currently not scalable, and existing DLAs rely on excitation by free-space lasers, which require the use of bulky optics.

Scaling DLAs to energies useful for applications and making them smaller requires a transition to integrated photonics, where light can be coupled on-chip, routed through waveguides, split into large networks, and manipulated with the precision afforded by nanofabrication techniques.

However, integration is challenging owing to scattering and reflections from the sub-wavelength features that manipulate the light to generate the accelerating fields. Moreover, traditional approaches in photonics design are often based on tuning simple geometric parameters, and produce structures that are highly sensitive to fabrication and wavelength offsets.

"To address these issues, we took advantage of powerful photonic inverse design techniques that use gradient-based optimization to explore the complete design space of a problem," explains Sapra.

"With this inverse design approach, we specified the waveguide stack, design region, and instructions to optimize for particle acceleration, and obtained a robust, very nonintuitive design that met all our desired specifications."

Following the input from the inverse design process, the researchers fabricated the optimized structure on a silicon-on-insulator wafer. Light from a pulsed laser was coupled by a grating to the fundamental mode of an integrated waveguide; this excited mode in turn excited the accelerator structure, which produced near fields that accelerated the electron beam, supplied by a scanning transmission electron microscope. The maximal energy gain was $0.915 \mathrm{keV}$ over $30 \mu \mathrm{m}$, which corresponds to a total acceleration gradient of $30.5 \mathrm{MeV}$ per meter.

"In this work, we showed dielectric laser acceleration over a single stage of acceleration of 30 microns; around a thousand such stages would be needed for an on-chip MeV-scale accelerator," says Sapra. "As next steps, we will improve the efficiency of our system to increase the amount of acceleration per stage and we will cascade additional stages to reach larger energies."

A MeV-scale accelerator operating at low currents could be used for localized tumour irradiation for cancer therapies, or as a table-top $\mathrm{X}$-ray source for imaging in industry and research. "On-chip not only promises smaller accelerators, but also greater cost-effectiveness due to the nanofabrication processes used to produce them," concludes Sapra. "With this research, we aim to democratize accelerator technologies to a wide range of applications."

Giulia Pacchioni

ORIGINAL ARTICLE Sapra, N. V. et al. On-chip integrated laser-driven particle accelerator. Science 367, 79-83 (2020) 